\title{
The effects of COVID-19 pandemic on emergency anterior abdominal wall hernia surgery: is it safe to postpone elective hernia surgery in the pandemic?
}

\author{
Umit Turan $^{1}$ (D) Ahmet Baris Dirim ${ }^{1}$ (D)
}

Received: 23 June 2021 / Accepted: 28 September 2021 / Published online: 4 October 2021

○) Springer-Verlag GmbH Germany, part of Springer Nature 2021

\begin{abstract}
Background The aim of this study was to investigate the effects of the COVID-19 pandemic on emergency anterior abdominal wall hernia surgeries (EAAWHS) by comparing the pandemic period with the control period a year ago and to share our experiences in the pandemic period.

Methods This single-center retrospective cohort study included all patients who underwent EAAWHS during the pandemic (from 11 March 2020 to 25 January 2021) and control period (1 year before the same period, from 11 March 2019 to 25 January 2020). Demographic data, preoperative clinical and pathological parameters, intraoperative findings and postoperative complications secondary to operation and COVID-19 infection, length of intensive care and hospital stay of patients were recorded, and the pandemic and control groups were compared.

Results Of the 87 patients who underwent anterior abdominal wall hernia surgery during the COVID-19 pandemic, 41 (47.1\%) were operated emergently and $46(52.9 \%)$ were operated electively. Of the 485 patients who underwent anterior abdominal wall hernia surgery during the control period, $24(4.95 \%)$ were operated emergently and $461(95.05 \%)$ were operated electively. The decrease in the number of elective operations and the increase in the number of emergency operations were significant during the pandemic $(p<0.001)$. There was a decrease in the number of emergency inguinal hernia operations and an increase in the number of emergency ventral (incisional, umbilical) hernia operations during the pandemic period compared to the control period $(p<0.05)$. The mortality rates were similar $(8.3$ vs. $9.8 \%, p>0.05)$ in both periods. Conclusion Despite the increase in the number of EAAWHS during the COVID-19 pandemic, there was no significant difference in mortality and morbidity rates. EAAWHS can be performed safely during the pandemic by taken necessary and adequate precautions.
\end{abstract}

Keywords COVID-19 $\cdot$ Emergency $\cdot$ Hernia $\cdot$ Morbidity $\cdot$ Mortality $\cdot$ Surgery

\section{Introduction}

The World Health Organization (WHO) declared COVID-19 infection a pandemic on March 11, 2020 [1]. On the same date, the first case of COVID-19 infection was reported in Turkey, since then restrictions have been implemented in

Ahmet Baris Dirim

drbarisdirim@yahoo.com

Umit Turan

turanumit@gmail.com

1 Department of General Surgery, Adana City Research and Training Center, Saglik Bilimleri University, Adana, Turkey the whole country to reduce contact and transmission in line with the decision taken by the Ministry of Health and the Scientific Board. From the date of 11 March 2020, elective surgical operations have been postponed throughout the country, and only oncological and emergency operations had been performed.

During the COVID pandemic that redistribution of all healthcare personnel including surgeons to effectively manage the surge in patient numbers required most hospital to restrict elective surgery and outpatient admissions due to limited hospital beds and equipment which were being used at maximum capacity for COVID-19 patients. The mortality rate of COVID-19 infection reported by WHO is approximately $3 \%$. The mortality rates increase even more in the elderly patient population, hypertensive and 
immunosuppressed patients, those undergoing surgery, and those with cancer [2-4].

Anterior abdominal wall hernia surgery is among the most frequently performed operations in general surgery practice. Postponing elective hernia operations has raised concerns that the number of patients presenting with incarceration and strangulation emergencies might increase. A pre-pandemic study reported the long-term probability of requiring emergency hernia repair as $0.2 \%$ for male patients with minimally symptomatic inguinal hernia followed up non-operatively (watchful waiting) [5]. Moreover, although Lima et al., in their letter to the editor published in the early period of the pandemic, reported no increase but a decrease in the number of elective hernia surgeries and a significant decrease in the number of emergency consultations for hernia with the cessation of elective hernia operations, there are also publications indicating no change in the hospitalization rates from the emergency department during the pandemic [6-8].

High mortality rates have been reported in patients with perioperative COVID-19 infection [3]. Although East et al. reported that a manual reduction of hernia under analgesia/ sedation (TAXIS) could be safely performed on selected patients during the pandemic period, some strangulated incarcerated hernias should be immediately operated [9]. Studies in the non-pandemic period have reported the mortality rate of EAAWHS between 1.5 and $4.5 \%$, while morbidity rates up to $38.8 \%$ have been reported and the most important predictive factor for mortality has been shown to be intestinal infarction [10-12]. COVID-19 infection is expected to increase mortality and morbidity rates during the pandemic in these patients requiring to be operated urgently [3]. Management of these patients in terms of COVID-19 infection is of great importance to reduce mortality and morbidity rates.

The aim of this study was to investigate the effects of COVID-19 infection on EAAWHS by comparing the outcomes of patients who underwent EAAWHS during the pandemic with those who underwent the same surgery in the non-pandemic control period a year ago. Furthermore, we aimed to share our precautions and experiences during the pandemic.

\section{Materials and methods}

This single-center retrospective study included the period between 11 March 2020 and 25 January 2021 as the pandemic period and between 11 March 2019 and 25 January 2020 as the control period. The study included all patients who were operated for anterior abdominal wall hernia in the General Surgery Clinic of the University of Health Sciences Adana City Training and Research Hospital, which serves pandemic patients with 1450 service beds and 300 intensive care beds. Patients who underwent hernia repair in addition to the primary operation and those who were operated for eventration or evisceration were excluded from the study.

The data of only emergency operated patients were analyzed by reviewing the hospital system. Patients presenting with symptoms of mechanical intestinal obstruction, incarceration and/or strangulation were urgently operated, while patients with a history of incarceration in the pandemic period or severe pain who did not have an indication for emergency surgery were operated electively. Demographic data (age, gender), ASA (American Society of Anaesthesiologists) score, comorbidity status, hernia type, whether resection was performed, use of mesh, COVID-19 reverse transcription polymerase chain reaction (RT-PCR) test results, preoperative and postoperative chest computed tomography scores according to the CO-RADS classification (A Categorical CT Assessment Scheme for Patients Suspected of Having COVID-19-Definition and Evaluation), the severity of postoperative surgical morbidities were graded according to the Clavien-Dindo Classification, length of hospital stay (service and intensive care unit), and mortality status of patients were recorded $[13,14]$. The results of hospital readmission, hospitalization, and mortality due to COVID19 infection within the first 30 days after discharge were also evaluated. COVID-19 infection status of the general surgery physician team was also recorded.

Preoperative and postoperative COVID-19 infection status of patients during the pandemic period was evaluated according to the classification proposed by Cano-Valderrama et al. [15]. COVID-19 status was classified as follows: negative (negative reverse transcription polymerase chain reaction (RT-PCR) for SARS-CoV-2), not-suspected (not tested for SARS-CoV-2 and no clinical symptoms), suspected (clinical symptoms of COVID without SARS-CoV-2 test or with negative RT-PCR), and positive (positive RT-PCR for SARS-CoV-2). Using separate elevators and transfer routes for suspected or positive patients, they were admitted to the service and intensive care units reserved for COVID-19 patients and operated in COVID-19 operating rooms.

Based on the evaluation of clinical, laboratory and imaging tests at emergency admission, patients who were eligible for TAXIS procedure were attempted to be treated with reduction under analgesia and sedation, and those who achieved reduction were observed in the ward for 24-48 $\mathrm{h}$. Patients whose symptoms regressed and who tolerated the oral intake were planned to be discharged by scheduling an elective operation. Those with a failed or successful reduction in the emergency department and progressive symptoms during the ward follow-up were urgently operated.

All patients were followed up postoperatively in a singleroom inpatient service unit, and they were invited for outpatient follow-up examinations by planning their discharge 
as soon as possible with and without drain. In case that the healthcare personnel was infected with COVID-19, they immediately took leave with isolation rules, and then started working at the hospital again if they were symptom-free following 10 days of quarantine.

\section{Statistical analysis}

The statistical analysis was performed using SPSS 25 (SPSS Inc). Chi-square test was used to compare categorical variables between groups. The Shapiro-Wilk normality test was used to determine whether the continuous variables had a normal distribution. The non-parametric Mann-Whitney $U$ test was used to compare the continuous variables without a normal distribution. Student $t$ test was used, if continous variables have normal distribution. A $p$ value $<0.05$ was considered statistically significant.

Research is being reported in line with the STROCCS criteria [16]. This study was registered in the Chinese Clinical Trial Registry (Code: ChiCTR2100048866).

\section{Results}

Of the 87 patients who were operated for anterior abdominal wall hernia during the COVID-19 pandemic, 41 (47.1\%) were operated urgently and 46 patients (52.9\%) were operated electively. Of the 485 patients who were operated during the control period, 24 (4.95\%) were operated urgently and $461(95.05 \%)$ were operated electively. The comparison of the two groups showed that the decrease in the number of elective operations and the increase in the number of emergency operations were statistically significant during the pandemic $(p<0.001)$.

From this point of our article, the outcomes of patients who underwent EAAWHS during the pandemic and control period will be given comparatively. Of the patients who were operated urgently during the pandemic, 26 (63.4\%) were female and 15 (36.6\%) were male, with a mean age of $63.26 \pm 16.20$ years. Of the patients who were operated during the control period, $6(25 \%)$ were female and 18 (75\%) were male, with a mean age of $54.54 \pm 22.54$ years. There was a more than twofold increase in the number of female patients operated during the pandemic compared to the control period, while there was a decrease by half in the number of male patients, which was statistically significant $(p=0.003)$.

The analysis of the hernia types of the emergency operated patients revealed that $10(24.4 \%)$ patients were operated for inguinal hernia, 12 (29.3\%) patients for umbilical hernia, 18 (43.9\%) patients for incisional hernia, and 1 (2.4\%) patient was operated for femoral hernia during the pandemic. In the control period, 13 (54.2\%) patients were operated for inguinal hernia, 4 (16.7\%) patients for umbilical hernia, 5 (20.8\%) patients for incisional hernia, and $2(8.3 \%)$ patients were operated for femoral hernia. The comparison of the two groups by hernia types showed a statistically significant difference $(p=0.042)$. There was a proportionate decrease in the number of inguinal hernias and an increase in the number of ventral (incisional, umbilical) hernias during the pandemic.

The most common comorbidities observed in patients operated during the pandemic and control period were diabetes mellitus and hypertension (19.5 vs. $20.8 \%, 22$ vs. $16.7 \%$, respectively). The evaluation of the ASA scores regarding these comorbidities showed that most patients in both groups had an ASA score of 3, with no difference between the two groups $(p>0.05)$. The data on the demographic and clinical status of patients operated in the pandemic and control period are given in Table 1.

Intraabdominal organ resection was performed on 14 (34.1\%) patients during the pandemic and on $10(41.7 \%)$ patients during the control period, with no statistically significant difference between the two groups $(p>0.05)$. The small intestine was the most frequently resected organ in both groups. The comparison of the groups by the use of mesh in emergency hernia surgery revealed that mesh was used for $23(56.1 \%)$ patients operated during the pandemic and for $12(50 \%)$ patients operated in the control period, with no difference between the two groups $(p>0.05)$.

When complications were graded according to the Clavien-Dindo classification, the rates of grade 3-5

Table 1 Comparative demographic and clinical characteristics of patients who underwent emergency anterior abdominal wall hernia surgery during the pandemic and control period

\begin{tabular}{llll}
\hline & Pandemic $n \%$ & Control $n \%$ & $p$ \\
\hline $\begin{array}{l}\text { Age mean year } \pm \text { SD } \\
\text { Gender }\end{array}$ & $63.26 \pm 16.20$ & $54.54 \pm 22.54$ & 0.07 \\
Female & & & \\
Male & $26(63.4)$ & $6(25)$ & 0.003 \\
Hernia type & $15(36.6)$ & $18(75)$ & \\
Inguinal & & & \\
Umbilical & $10(24.4)$ & $13(54.2)$ & 0.042 \\
Insicional & $12(29.3)$ & $4(16.7)$ & \\
Femoral & $18(43.9)$ & $5(20.8)$ & \\
Comorbidities & $1(2.4)$ & $2(8.3)$ & \\
Hypertansion & & & \\
Diabetes mellitus & $9(21.6)$ & $4(16.7)$ & \\
Cardiac & $8(19.2)$ & $5(20.8)$ & \\
Malignancy & $12(29.3)$ & $4(16.7)$ & \\
Pulmonary & $7(16.8)$ & $1(4.1)$ & \\
ASA score & $2(4.8)$ & $1(4.1)$ & \\
ASA-1 & & & \\
ASA-2 & $8(19.2)$ & $4(16.7)$ & 0.45 \\
ASA-3 & $13(31.7)$ & $7(29.1)$ & \\
ASA-4 & $17(41.4)$ & $8(33.3)$ & \\
\hline
\end{tabular}


postoperative morbidities were higher in the control period compared to the pandemic (16.7 vs. $9.8 \%, p=0.413)$, with no statistically significant difference $(p>0.05)$. The postoperative outcomes of patients are given in Table 2.

For patients operated during the pandemic and control period, the mean length of intensive care stay was $1.73 \pm 3.18$ vs. $1.20 \pm 2.16$ days and the mean length of hospital stay was $6.68 \pm 7.78$ vs. $6.79 \pm 8.15$ days. The comparison of the two groups by the length of stay revealed that the length of intensive care stay was longer during the pandemic but was not statistically significant $(p>0.05)$.

During the pandemic, $1(2.4 \%)$ patient was preoperatively suspected of having COVID-19 infection, while 3 (7.3\%) patients were postoperatively suspected of having COVID19 infection, and $1(2.4 \%)$ patient had a positive RT-PCR test result postoperatively. The patient with a positive test result was operated for incisional hernia without organ resection and was discharged after being followed up in the pandemic service. Three $(7.3 \%)$ of the patients who were operated during the pandemic were readmitted to the hospital within the first 30 days post-operation due to suspected COVID-19 infection; however, the RT-PCR test results of these patients were negative. Of the patients with a negative RT-PCR test result, 1 presented as an outpatient, while 2 were referred to our hospital from the intensive care unit of another hospitals. During the study period, 8 individuals (25\%) from the physician team of the General Surgery Clinic had a positive RT-PCR test result. COVID-19 status of patients who underwent EAAWHS during the pandemic period is presented in Table 3. Patients with preoperatively and postoperatively suspected COVID-19 and a positive RT-PCR test result were not the same patients.

Death was observed $4(9.8 \%)$ vs $2(8.3 \%)$ in the pandemic and control group, with no difference between the two groups $(p>0.05)$. One of the patients who died during the pandemic had severe heart failure and died of cardiac arrhythmia in the postoperative third hour. The other 3
Table 3 Preoperative and postoperative COVID-19 status of patients with anterior abdominal wall hernia emergency surgery during the pandemic

\begin{tabular}{lll}
\hline & Preoperative $n \%$ & Postoperative $n \%$ \\
\hline Negative & $2(4.8)$ & $14(34.1)$ \\
Not suspected & $38(92.6)$ & $24(58.5)$ \\
Suspected & $1(2.4)$ & $3(7.3)$ \\
Positive & 0 & $1(2.4)$ \\
\hline
\end{tabular}

patients died of pulmonary complications. The RT-PCR test results of these 3 patients who died of pulmonary complications were negative. One of them was operated for an umbilical hernia, underwent small bowel resection for necrosis, could not be disconnected from the mechanical ventilator postoperatively, died on postoperative day 16 , and the chest CT was consistent with CO-RADS 4 . The other 2 patients who died, readmitted to another center with pulmonary symptoms during the one month of follow-up period after discharge and were transferred to our hospital. Their chest CT's were consistent with CO-RADS 3 and 4. Two patients who died in the control period required small bowel resection for necrosis, and an ileostomy was created for these patients due to intraabdominal contamination. 89-year-old patient developed postoperative neurological symptoms and was found to have cerebral meningioma on post office work up, the other patient was 45-year-old and died of intraabdominal sepsis. The general characteristics of the patients who died are presented in Table 4.

\section{Discussion}

Although postponing elective hernia operations during the pandemic has raised concerns that the number of EAAWHS may increase due to incarceration or strangulation, recent
Table 2 Comparison of postoperative results during the pandemic and control period

\begin{tabular}{llll}
\hline & Pandemic $n \%$ & Control $n \%$ & $p$ \\
\hline Resection & & & \\
Yes & $14(34.1)$ & $10(41.6)$ & 0.43 \\
No & $27(65.8)$ & $14(58.3)$ & \\
Mesh & & & \\
Yes & $23(56)$ & $12(50)$ & 0.54 \\
No & $18(44)$ & $12(50)$ & \\
Clavien-Dindo classification, grade (III-V) & $4(9.75)$ & $4(16.66)$ & 0.41 \\
Postoperative complication & $12(29.2)$ & $7(29.16)$ & 0.63 \\
Length of hospital stay, mean day \pm SD & $6.68 \pm 7.78$ & $6.79 \pm 8.15$ & 0.72 \\
Length of ICU stay mean day \pm SD & $1.73 \pm 3.18$ & $1.20 \pm 2.16$ & 0.39 \\
Death status & & & \\
Yes & $4(9.7)$ & $2(8.3)$ & 0.84 \\
No & $37(90.3)$ & $22(91.7)$ & \\
\hline
\end{tabular}


Table 4 Characteristics of patients who underwent emergency anterior abdominal wall hernia surgery during the pandemic and control period with postoperative mortality

\begin{tabular}{lllllllll}
\hline & Age & Gender & ASA & Resection & Covid status & Comorbidity & Complication & Surgery type (Hernia Repair) \\
\hline Pandemic & & & & & & & & \\
1 & 67 & F & 2 & - & Suspected & Lymphoma & Pulmonary & Insicional \\
2 & 82 & F & 3 & Small bowel & Suspected & Cardiac & Pulmonary & Umbilical \\
3 & 55 & M & 2 & - & Suspected & - & - & Umbilical \\
4 & 78 & M & 4 & - & Not suspected & Cardiac & Cardiac & Inguinal \\
Control & & & & & & & \\
1 & 89 & F & 4 & Small bowel & - & Malignancy & Wound infection & Femoral ileostomy \\
2 & 45 & M & 2 & Small bowel & - & - & Intraabdominal sepsis & Inguinal ileostomy \\
\hline
\end{tabular}

studies have reported a decrease in the number of patients $[21,22]$. In our study, 41 patients were operated emergently during the pandemic, while 24 patients were operated during the control period. We believe that the reason for the increase in the number of our EAAWHS patients during the pandemic is due to the fact that our hospital is a tertiary referral center serving a population of 4.5 million and elective hernia surgeries have been restricted during the pandemic.

The comparison between the pandemic and control period in this study showed a difference in the rate of operated hernia types, with a statistical significance. While there was a decrease by approximately half in the number of inguinal hernias operated urgently during the pandemic, there was approximately a twofold increase in the number of ventral hernias (incisional, umbilical). In a non-pandemic period study of 1358 patients by Kokotovic et al. analyzing "watchful waiting" in ventral hernias reported the rate of ventral hernia patients requiring emergency operation as $4 \%$ within 5 years [17]. A similar study by Martin et al. reported this rate as $8.3 \%$ [18]. The rate of emergency repair has been reported to be $0.2 \%$ when male patients with minimally symptomatic inguinal hernia are not operated [5]. As reported by previous studies, the possibility of requiring emergency surgery in the "watchful waiting" approach to ventral hernias is much higher than inguinal hernias. The increase in the number of urgent ventral hernias in our study is considered to be due to the restriction of elective operations. Therefore, we think that the reliability of the "watchful waiting" approach to ventral hernias should be questioned.

During the pandemic period, non-operative treatment approaches have been recommended for selected patients with general surgical emergencies such as acute cholecystitis and acute appendicitis [19, 20]. Non-operative treatment approaches cannot be used in incarcerated/strangulated hernias which leads mechanical obstruction and tissue ischemia. For this reason, the rate of emergency hernia surgery may increase in emergent general surgery operations. In shortterm studies with a low number of EAAWHS cases, it was reported that EAAWHS was decreased during the pandemic period compared to the control period. [21, 22]. Oscar CanoValderemma et al. reported a decrease in the number of general surgery emergency groups but an increased number of incarcerated hernia cases [15].

Previous studies have reported the mortality rate following EAAWHS in the non-pandemic period between 1.5 and $4.5 \%[8,9]$. In our study, the mortality rate was $8.3 \%$ during the control period, while it was $9.8 \%$ during the pandemic. We think that the reason for the higher mortality rate in our study compared to previous studies is due to the fact that our hospital is a tertiary referral hospital to which elderly patients with a higher prevalence of comorbidities are referred. In our study supporting this opinion, the mean age of patients operated during the pandemic was high $(63.26 \pm 16.20$ years $)$ and $75 \%$ of these patients had at least one comorbid disease. The comparison between two groups by gender showed that there was a more than twofold increase in the number of female patients operated during the pandemic compared to those operated in the control period, while there was a decrease by half in the number of male patients. We could not explain the reason for such a difference in gender in our study patient population.

When complications were graded according to the Clavien-Dindo classification, the rates of grade 3-5 postoperative complications were higher in the control period compared to the pandemic, though not statistically significant (16.7 vs. 9.8\%). Most of the patients undergoing EAAWHS have advanced age and a high prevalence of comorbidities [8]. These are the risk factors for mortality from COVID-19 infection. The mortality of emergency surgery, on the other hand, is higher in patients with COVID-19 infection. In their studies of 22 patients with COVID-19 infection who underwent emergency abdominal surgery, Rasslan et al. reported the mortality rate as $31.8 \%$ [23]. Our study demonstrated that the pandemic did not increase the mortality and morbidity rates for EAAWHS in the general surgery clinic. The reason of no increase in mortality during the pandemic is that we have 
been able to minimize the cross transmission of the virus from patient to patient and from patient to healthcare staff depending on the precautions we have taken.

The evaluation of the mean length of intensive care $(1.73 \pm 3.18$ vs. $1.20 \pm 2.16$ days $)$ and hospital stay $(6.68 \pm 7.78$ vs. $6.79 \pm 8.15$ days) during the pandemic and control periods revealed that though not statistically significant, the length of intensive care stay was longer during the pandemic period despite our efforts to discharge patients as early as possible to reduce virus transmission during the pandemic period. We are of the opinion that this is due to older age and comorbidities of patients operated in the pandemic compared to those operated in the control period.

In addition, the analysis of the 4 patients who died during the COVID-19 pandemic revealed that 1 of these patients died of cardiac complications and 3 of them died of pulmonary complications. The COVID-19 RT-PCR results of these 3 patients were negative. While 1 (2.4\%) of the patients included in our study had a positive RT-PCR test result postoperatively, 8 (25\%) of the physician team working in the general surgery clinic had a positive RT-PCR test result in the same period, and 1 from the physician team was hospitalized due to his comorbidities. As a similar result of our study, Gallego et al. reported that 12 (24\%) of the general surgeons in their study were infected [24]. We thought that the reason why the surgical team was infected by $25 \%$ despite the strict contact isolation precautions, may be due to the aggressiveness of the virus and the fact that the surgeons worked in the COVID-19 service and intensive care units.

During the pandemic period, 3 (7.3\%) patients were readmitted to the hospital within the first 30 days after discharge upon the suspicion of COVID-19 infection; however, none of these patients had a positive RT-PCR test result. One patient was followed up as an outpatient, while the other 2 patients who were hospitalized for respiratory distress in the intensive care unit of another centers within 3 weeks after discharge were referred to us and died. Patients could also come into contact with virus in their normal lives, and we interpret the low number of patients presented with the suspicion of COVID-19 in the early postoperative period as an indicator of how effective the virus transmission measures were.

The limitations of this study are its single-center retrospective nature and small sample size. However, we are of the opinion that the demonstration of the reliability of surgery during the COVID-19 pandemic with the protective measures, showing the increase in the number of EAAWHS and the proportional change in hernia types are valuable outcomes. Furthermore, our work is the first study including such a long period for EAAWHS during the pandemic as well as presenting the data of such a large number of patients.

\section{Conclusion}

In conclusion, our study demonstrated an increase in the number of emergency anterior abdominal wall hernia surgeries and a proportional change in hernia types during the pandemic but no difference in terms of mortality and morbidity. While the rate of requiring emergency surgery for inguinal hernias was reduced by half with the watchful waiting approach and TAXIS procedure during the pandemic, there was a twofold increase in the number of ventral hernias. These results should be evaluated with larger patient series. We are of the opinion that EAAWHS can be performed safely during the pandemic by taking the necessary precautions.

Author contributions UT: design of the study, interpretation, critical revision of the paper. ABD: acquisition of data, data analysis.

Funding The Authors declare that this research did not receive any specific grant from funding agencies.

Availability of data and material The data are available from the authors on reasonable request.

\section{Declarations}

Conflict of interest The authors declare that they have no competing interests.

Ethical approval All procedures performed in the study involving human participants were in accordance with the ethical standards of the institutional and national research committee and with the 1964 Helsinki declaration and its later amendments or comparable ethical standards. This research was approved by the Institutional Review Board by the number 79/1373 and national research committee by the number 2021_02_05T10_57_20.

Consent for publication Informed consent was obtained from all individual participants of the study.

\section{References}

1. World Health Organization (WHO) COVID-19 Dashboard. https:// covid19.who.int/region/euro/country/it.

2. Yang K, Sheng Y, Huang C, Jin Y, Xiong N, Jiang K, et al. Clinical characteristics, outcomes, and risk factors for mortality in patients with cancer and COVID-19 in Hubei, China: a multicentre, retrospective, cohort study. Lancet Oncol. 2020;2(7):904-13.

3. Nepogodiev D, Glasbey J, Li E, Omar O, Simoes J, Abbott T, et al. Mortality and pulmonary complications in patients undergoing surgery with perioperative SARS-CoV-2 infection: an international cohort study. Lancet. 2020. https://doi.org/10.1016/S01406736(20)31182-X.

4. Kuderer NM, Choueiri TK, Shah DP, Shyr Y, Rubinstein SM, Rivera DR, et al. Clinical impact of COVID-19 on patients with cancer (CCC19): a cohort study. Lancet. 2020. https://doi.org/10. 1016/S0140-6736(20)31187-9. 
5. Fitzgibbons RJ Jr, Ramanan B, Arya S, Turner SA, Li X, Gibbs JO, Reda DJ, Investigators of the Original Trial. Long-term results of a randomized controlled trial of a nonoperative strategy (watchful waiting) for men with minimally symptomatic inguinal hernias. Ann Surg. 2013;258(3):508-15.

6. Lima DL, Pereira X, Dos Santos DC, Camacho D, Malcher F. Where are the hernias? A paradoxical decrease in emergency hernia surgery during COVID-19 pandemic. Hernia. 2020;24(5):1141-2.

7. Jefery MM, D'Onofrio G, Paek H, Platts-Mills TF, Soares WE, Hoppe JA, et al. Trends in emergency department visits and hospital admissions in health care systems in 5 States in the first months of the COVID-19 pandemic in the US. JAMA Intern Med. 2020;180(10):1328-33.

8. Kim HS, Cruz DS, Conrardy MJ, Gandhi KR, Seltzer JA, Loftus TM, et al. Emergency department visits for serious diagnoses during the COVID19 pandemic. Acad Emerg Med. 2020;27:910.

9. East B, Pawlak M, de Beaux AC. A manual reduction of hernia under analgesia/sedation (Taxis) in the acute inguinal hernia: a useful technique in COVID-19 times to reduce the need for emergency surgery-a literature review. Hernia. 2020. https://doi.org/10. 1007/s10029-020-02227-1.

10. Kartal A, Çitgez B, Besler E, Yetkin SG, Uludağ M, Akgün İE, Özşahin H, Mihmanlı M. Our experience of incarcerated inguinal hernias. Med Bull Şişli Etfal Hospital. 2014;48(4):287-90. https:// doi.org/10.5350/SEMB.20140810102931.

11. Russell TB, Elberm H. Emergency hernia surgery at a highvolume tertiary centre: a 3-year experience. ANZ J Surg. 2021;91(4):622-6.

12. Martínez-Serrano MA, Pereira JA, Sancho J, Argudo N, LópezCano M, Grande L. Specific improvement measures to reduce complications and mortality after urgent surgery in complicated abdominal wall hernia. Hernia. 2012;16(2):171-7.

13. Prokop M, van Everdingen W, van Rees VT, et al. CO-RADS - a categorical CT assessment scheme for patients with suspected COVID-19: definition and evaluation. Radiology. 2020;1:201473.

14. Dindo D, Demartines N, Clavien PA. Classifcation of surgical complications: a new proposal with evaluation in a cohort of 6336 patients and results of a survey. Ann Surg. 2004;240(2):205-13.

15. Cano-Valderrama O, Morales X, Ferrigni CJ, Martín-Antona E, Turrado V, García A, et al. Acute Care Surgery during the COVID-19 pandemic in Spain: changes in volume, causes and complications. A multicentre retrospective cohort study. Int J Surg. 2020;80:157-61.

16. Agha RA, Abdall-Razak A, Crossley E, Dowlut N, Iosifidis C, Mathew G. The STROCSS 2019 guideline: strengthening the reporting of cohort studies in surgery. Int J Surg. 2019;72:156-65. https://doi.org/10.1016/j.ijsu.2017.08.586.

17. Kokotovic D, Sjølander H, Gögenur I, Helgstrand F. Watchful waiting as a treatment strategy for patients with a ventral hernia appears to be safe. Hernia. 2016;20(2):281-7.

18. Martin AC, Lyons NB, Bernardi K, Holihan JL, Cherla DV, Flores JR, Huang L, Milton A, Shah P, Kao LS, Ko TC, Liang MK. Expectant management of patients with ventral hernias: 3 years of follow-up. World J Surg. 2020;44(8):2572-9.

19. Çiyiltepe H, Yıldırım G, Fersahoğlu MM, Aydın MT, Özcabı Y, Bulut NE, et al. Clinical approach to patients admitted to the emergency room due to acute cholecystitis during the COVID-19 pandemic and percutaneous cholecystostomy experience. Ulus Travma Acil Cerrahi Derg. 2021;27(1):34-42.

20. Collard M, Lakkis Z, Loriau J, et al. Antibiotics alone as an alternative to appendectomy for uncomplicated acute appendicitis in adults: changes in treatment modalities related to the COVID-19 health crisis. J Vis Surg. 2020. https://doi.org/10.1016/j.jviscsurg. 2020.04.014.

21. Surek A, Ferahman S, Gemici E, Dural AC, Donmez T, Karabulut M. Effects of COVID-19 pandemic on general surgical emergencies: are some emergencies really urgent? Level 1 trauma center experience. Eur J Trauma Emerg Surg. 2020. https://doi.org/10. 1007/s00068-020-01534-7.

22. Abu Shakra I, Bez M, Ganam S, Francis R, Muati A, Bickel A, Merei F, Talmi Z, Kamal K, Kakiashvili E. The volume of general surgery emergency cases in a government hospital during the COVID-19 pandemic and two other periods: a comparative, retrospective study. BMC Surg. 2021;21(1):119.

23. Rasslan R, Dos Santos JP, Menegozzo CAM, Pezzano AVA, Lunardeli HS, Dos Santos MJ, Utiyama EM, Damous SHB. Outcomes after emergency abdominal surgery in COVID-19 patients at a referral center in Brazil. Updates Surg. 2021;73(2):763-8.

24. Gallego MA, de las Casas SG, Migueláñez IP, et al. Impacto de la pandemia por SARS-CoV-2 sobre la actividad y profesionales de un Servicio de Cirugía General y del Aparato Digestivo en un hospital terciario. Cir Esp. 2020;98:320-7. https://doi.org/10. 1016/j.ciresp.2020.04.001. 